\title{
Selection of AVP-Shortage Patients as Candidates for Low-Dose Oral Desmopressin Administration
}

This article was published in the following Dove Press journal:

Research and Reports in Urology

\author{
Takumi Takeuchi (D) \\ Kazuki Maki \\ Yumiko Okuno' \\ Mami Hattori-Kato ${ }^{2}$ \\ Koji Mikami' \\ 'Department of Urology, Japan \\ Organization of Occupational Health and \\ Safety, Kanto Rosai Hospital, Nakahara- \\ ku, Kawasaki, Japan; ${ }^{2}$ Department of \\ Urology, Tokyo Teishin Hospital, \\ Chiyoda-ku, Tokyo I02-8798, Japan
}

\begin{abstract}
Objective: We herein attempted to select male patients with an elevated nocturnal urinary frequency possibly due to a shortage of AVP. These patients may be good candidates for lowdose oral desmopressin administration.
\end{abstract}

Patients and Methods: Serum and spot urine osmolality, electrolytes, serum creatinine, casual blood glucose, plasma brain natriuretic polypeptide (BNP), and plasma AVP were measured at the same time in 97 elderly male patients with urinary symptoms under free water drinking.

Results: A binary plot of plasma AVP and serum osmolality indicated a region at which patients had relatively lower AVP considering higher serum osmolality. It was tentatively named the desmopressin region. Twenty out of 97 (20.6\%) patients were in the desmopressin region. Daily urine output did not exceed $3 \mathrm{~L}$ in any patient. Urine osmolality was slightly lower in patients in the desmopressin region. No significant differences were observed in urine volume, urinary frequency, or urination questionnaire scores between both groups.

Conclusion: AVP-shortage patients may be selected for treatment with oral desmopressin based on measurements of serum osmolality and plasma AVP.

Keywords: AVP, osmolality, desmopressin, urination

\section{Introduction}

Diabetes insipidus (DI) is characterized by the excretion of large volumes of hypotonic urine $(>50 \mathrm{~mL} / \mathrm{kg} / 24$ hours in adults) and thirst due to an impaired ability to concentrate urine, leading to uncontrolled diuresis, which may cause lifethreatening dehydration and electrolyte imbalances. ${ }^{1,2}$ There are three types of DI. Central DI is caused by the deficient secretion of the posterior pituitary antidiuretic hormone arginine vasopressin (AVP). The intracranial causes of central DI are neoplastic, vascular, trauma, inflammatory, infection, and idiopathic. ${ }^{1,2}$ Nephrogenic DI occurs when the response of renal tubules to absorb water is impaired due to resistance to the effects of AVP on its receptors. ${ }^{1,2}$ Gestational DI is transient during pregnancy due to an increase in the metabolism of vasopressin induced by placental cysteine aminopeptidase. ${ }^{1,3}$ Primary polydipsia is a disorder caused by excessive water intake and is often observed in patients with psychogenic issues. DI and primary polydipsia are collectively referred to as polyuria-polydipsia syndromes.

The AVP gene is in chromosome 20p13. Pre-pro-vasopressin, a peptide of 164 amino acids, is transcribed from the gene in magnocellular neurons in the supraoptic and paraventricular nuclei of the hypothalamus. It is converted to pro-vasopressin by
Correspondence: Takumi Takeuch Department of Urology, Japan

Organization of Occupational Health and Safety, Kanto Rosai Hospital, I-I Kizukisumiyoshi-Cho, Nakahara-ku, Kawasaki 2II-8510, Japan

Email takeuchit@abelia.ocn.ne.jp
Research and Reports in Urology 2021:13 25-30 
the removal of the signal peptide and N-linked glycosylation of copeptin. AVP, neurophysin II, and copeptin are produced by the further processing of pro-vasopressin followed by axonal transportation to the posterior pituitary. ${ }^{2}$

A water deprivation test followed by the administration of desmopressin (Deamino-8-D-AVP, the synthetic analogue of AVP, Minirinmelt ${ }^{\circledR}$ ) may theoretically differentiate between central and nephrogenic DI as well as primary polydipsia; however, overlapping test results sometimes affect the accuracy of the diagnosis because of the existence of partial central DI, partial nephrogenic DI, and chronic primary polydipsia. ${ }^{4}$ The measurement of plasma AVP levels in combination with the water deprivation test has also been suggested in order to distinguish between the various polyuria-polydipsia syndromes. ${ }^{5}$ DI patients need to drink a sufficient amount of fluids to avoid dangerous dehydration. Desmopressin is usually administered to treat central DI.

Desmopressin orally disintegrating tablets are administered to male patients with nocturia. ${ }^{6,7} \mathrm{We}$ herein attempted to select male patients with an elevated nocturnal urinary frequency possibly due to a shortage of AVP. These patients may be good candidates for low-dose oral desmopressin administration. Currently, the indication of low-dose desmopressin does not take the association of AVP with serum osmolality into consideration. It is therefore reasonable to administer it to patients who are lacking. Thus, we investigated the association between AVP and serum osmolality in elderly patients with urinary symptoms. It may be important to administer low-dose desmopressin after selecting patients with urinary symptoms lacking AVP.

Increased urine production has many causes, such as voluntarily increased water intake. When desmopressin is administered to patients who voluntarily drink a large amount of water, they have a higher risk of hyponatremia and cardiac failure due to excessive AVP function. Therefore, evaluation of AVP status is important even if urine production is similar.

\section{Patients and Methods}

This was a cross-sectional study. Patients visiting our hospital due to urinary symptoms were enrolled in this study. They were mainly diagnosed with benign prostatic hyperplasia and/or overactive bladder.

Serum and spot urine osmolality, electrolytes, serum creatinine, casual blood glucose, plasma brain natriuretic polypeptide (BNP), and plasma AVP were measured at the same time in 97 elderly male patients with urinary symptoms under free water drinking. The International Prostate Symptom Score (IPSS), Overactive Bladder Symptom Score (OABSS), and 24-hour frequency-volume charts at least twice were also evaluated. A 24-hour frequencyvolume chart states the time and volume of each void and the bedtime and waking time. The nocturnal urine volume was defined as the volume of voids between bedtime and waking time plus the first-morning void, while the first-morning void was regarded as a normal diurnal voiding episode. Data recorded from frequency-volume charts were averaged.

Blood and urine laboratory data, and IPSS and OABSS scores were measured during the visit to our hospital between January 2020 and August 2020. The 24-hour frequency-volume charts were recorded around laboratory data measurements. The information on comorbidities was collected from medical records.

\section{Statistical Analysis}

Age, prostate volume, biochemical data, urine volume, urinary frequency, and urination questionnaire scores were analyzed by the unpaired $t$-test. Rates of urination drug usages and co-morbidities were assessed by the twotailed chi-squared test.

\section{Ethics}

The present study was conducted in accordance with the Helsinki Declaration after approval of the Ethics Committee of Kanto Rosai Hospital (G2020-3). Written informed consent was received from all participants in the study. Written informed consent was received from the patients to have the case details published.

\section{Results}

Figure 1 shows a binary plot of plasma AVP and serum osmolality. The curves on the graph conveniently distinguished between central DI and renal DI/polydipsia. ${ }^{8}$ The region below this curve in addition to serum osmolality of more than $290 \mathrm{mOsm} / \mathrm{kg}$ was tentatively named the desmopressin region. Twenty out of $97(20.6 \%)$ patients were in the desmopressin region.

Table 1 shows the urination-related drugs already administered to and co-morbidities of patients. No significant differences were observed in the frequency of administered urinary drugs and existing co-morbidities in patients in the desmopressin and non-desmopressin regions. Table 2 shows data on biochemistry and urination. 


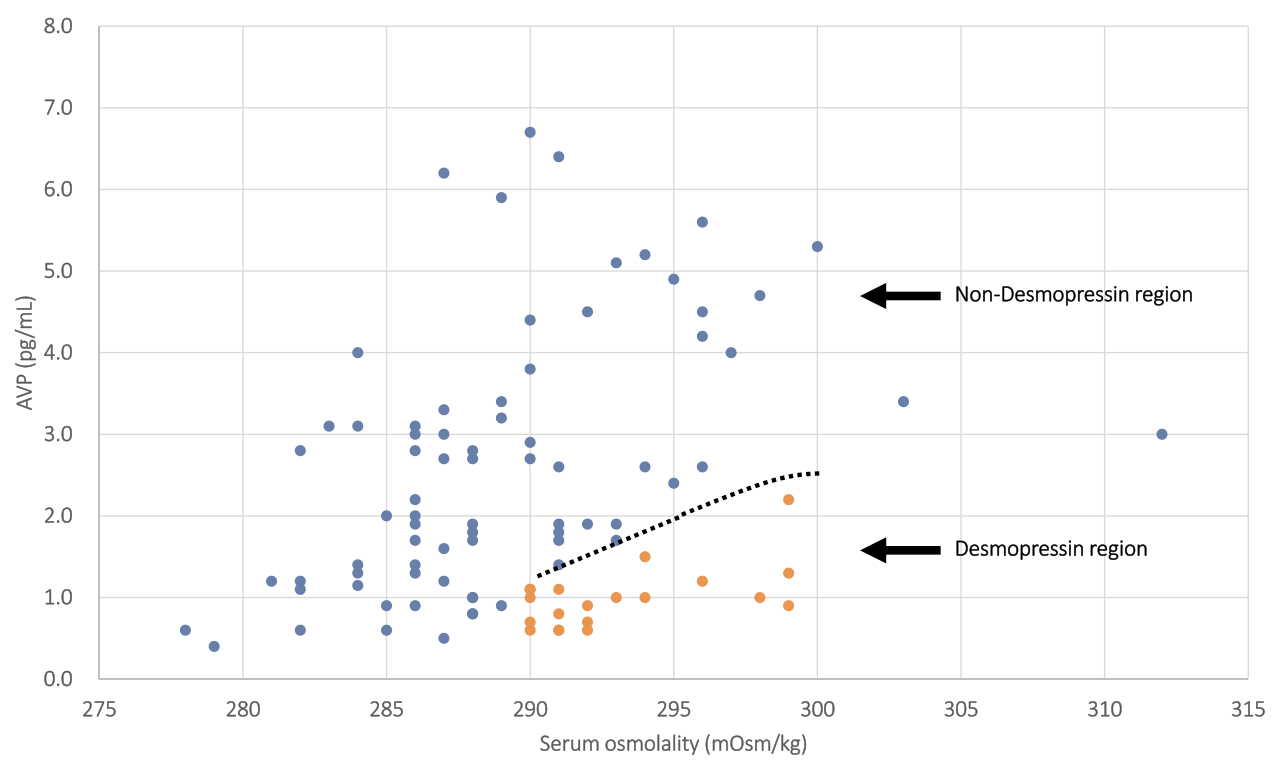

Figure I Binary plot of plasma AVP and serum osmolality in patients with urinary symptoms. Orange dots show AVP-shortage patients with elevated serum osmolality (desmopressin region), while blue dots are non-AVP-shortage patients (non-desmopressin region). The curve is the line that separates the two groups. Two cases showing AVP20.4 pg/mL, serum osmolality $290 \mathrm{mOsm} / \mathrm{kg}$ and AVP $10.0 \mathrm{pg} / \mathrm{mL}$, serum osmolality $301 \mathrm{mOsm} / \mathrm{kg}$ were excluded from plotting.

Table I Urinary Drugs Administered to and Co-Morbidities of Patients

\begin{tabular}{|c|c|c|c|}
\hline & Non-Desmopressin Region & Desmopressin Region & p-value \\
\hline \multicolumn{4}{|l|}{ Urination drugs } \\
\hline$\alpha \mathrm{l}$-adrenergic antagonist & $90.9(\%)$ & $90.0(\%)$ & 0.9006 \\
\hline PDE5 inhibitor & 0 & 5 & 0.5072 \\
\hline Anti-cholinergic & 11.7 & 15 & 0.9843 \\
\hline $5 \alpha$-reductase inhibitor & 59.7 & 50 & 0.595 \\
\hline$\beta 3$-adrenergic agonist & 5.2 & 0 & 0.6819 \\
\hline \multicolumn{4}{|l|}{ Co-morbidities } \\
\hline Hypertension & 42.9 (\%) & $60.0(\%)$ & 0.2635 \\
\hline Diabetes mellitus & 16.9 & 20 & 0.7439 \\
\hline Intracranial & 11.7 & 10 & 0.832 \\
\hline Cardiovascular & 24.7 & 25 & $0.976 I$ \\
\hline Liver disease & 1.3 & 5 & 0.877 \\
\hline Kidney disease & 2.6 & 15 & 0.0954 \\
\hline Lung disease & 2.6 & 0 & 0.4664 \\
\hline Orthopedic disease & 11.7 & 15 & 0.9843 \\
\hline Malignancy & 18.2 & 20 & 0.8522 \\
\hline
\end{tabular}

Notes: $\mathrm{N}=77$ and $\mathrm{N}=20$ for the non-desmopressin and desmopressin regions, respectively.

Daily urine output did not exceed $3 \mathrm{~L}$ in any patients. Plasma AVP was lower, while serum osmolality and serum sodium were higher in patients in the desmopressin region than in those in the non-desmopressin region. Furthermore, urine osmolality was slightly lower in patients in the desmopressin region. No significant differences were observed in urine volume, urinary frequency, or urination questionnaire scores between both groups.

\section{Case}

A male in his 90's was admitted to our hospital with nocturia despite being administered an $\alpha$-blocker, anti-cholinergic drug, and $\beta 3$ antagonist at a nearby urologic clinic. His prostate volume was $16 \mathrm{~g}$, residual urine was $18 \mathrm{~mL}$, and he had thirst. Serum $\mathrm{Na}$ was $140 \mathrm{mEq} / \mathrm{L}$, serum creatinine $0.89 \mathrm{mg} / \mathrm{dL}$, blood glucose level $126 \mathrm{mg} / \mathrm{dL}$, plasma BNP $45.0 \mathrm{pg} / \mathrm{mL}$, plasma AVP $1.0 \mathrm{pg} / \mathrm{mL}$, serum osmolality 298 
Table 2 Biochemistry and Urination Data of Patients

\begin{tabular}{|c|c|c|c|}
\hline & Non-Desmopressin Region & Desmopressin Region & p-value \\
\hline Age & $79.4 \pm 7.6$ & $81.5 \pm 7.9$ & 0.2791 \\
\hline Serum sodium (mEq/L) & $140.1 \pm 2.6$ & $142.1 \pm 2.0$ & 0.0302 \\
\hline Serum chloride (mEq/L) & $103.8 \pm 2.7$ & $104.9 \pm 2.2$ & 0.1011 \\
\hline Serum creatinine $(\mathrm{mg} / \mathrm{dL})$ & $1.00 \pm 0.34$ & $1.08 \pm 0.29$ & 0.2941 \\
\hline Casual blood glucose $(\mathrm{mg} / \mathrm{dL})$ & $122.5 \pm 35.9$ & $121.8 \pm 36.0$ & 0.936 \\
\hline Plasma BNP (pg/mL) & $51.6 \pm 76.1$ & $55.4 \pm 47.6$ & 0.8355 \\
\hline Plasma AVP (pg/mL) & $2.94 \pm 2.68$ & $1.00 \pm 0.38$ & 0.0018 \\
\hline Serum osmolality (mOsm/kg) & $289.1 \pm 5.6$ & $293.1 \pm 3.3$ & 0.0031 \\
\hline Urine osmolality (mOsm/kg) & $527.3 \pm 151.2$ & $456.1 \pm 166.4$ & 0.0693 \\
\hline Prostate volume $(\mathrm{mL})$ & $45.1 \pm 29.8$ & $36.4 \pm 18.5$ & 0.2172 \\
\hline Daytime urine volume $(\mathrm{mL})$ & $903 \pm 325$ & $950 \pm 343$ & 0.6077 \\
\hline Nocturnal urine volume $(\mathrm{mL})$ & $66 I \pm 36 I$ & $731 \pm 293$ & 0.4732 \\
\hline 24-hour urine volume (mL) & $|57| \pm 5 \mid 5$ & $1681 \pm 430$ & 0.4279 \\
\hline Nocturnal polyuria index (\%) & $40.5 \pm 14.2$ & $42.5 \pm 12.5$ & 0.6032 \\
\hline Daytime urinary frequency & $7.5 \pm 2.1$ & $7.3 \pm 1.7$ & 0.6853 \\
\hline Night time urinary frequency & $2.4 \pm 1.5$ & $2.8 \pm 2.0$ & 0.3832 \\
\hline 24-hour urinary frequency & $9.9 \pm 2.8$ & $10.1 \pm 3.1$ & 0.8416 \\
\hline IPSS-T & $10.8 \pm 6.7$ & $12.3 \pm 7.0$ & 0.386 \\
\hline IPSS-V & $5.4 \pm 4.8$ & $5.7 \pm 4.8$ & 0.7819 \\
\hline IPSS-S & $5.4 \pm 3.1$ & $6.1 \pm 3.2$ & 0.3818 \\
\hline IPSS-Q7 & $2.5 \pm 1.2$ & $2.6 \pm 1.4$ & 0.902 \\
\hline IPSS-QOL & $3.2 \pm 1.5$ & $3.6 \pm 1.5$ & 0.2741 \\
\hline OABSS & $5.2 \pm 3.5$ & $5.7 \pm 3.3$ & 0.5226 \\
\hline
\end{tabular}

Notes: $\mathrm{N}=77$ and $\mathrm{N}=20$ for the non-desmopressin and desmopressin regions, respectively. Nocturnal polyuria index: nocturnal urine volume divided by 24 -hour urine volume.

Abbreviations: IPSS-T, total IPSS score; IPSS-V, IPSS voiding score; IPSS-S, IPSS storage score.

$\mathrm{mOsm} / \mathrm{kg}$, and urine osmolality $542 \mathrm{mOsm} / \mathrm{kg}$. Frequencyvolume charts revealed a daily urine output of $2090 \mathrm{~mL}$, night time urine output rate $50.2 \%$, daily urination frequency 12 times, and night time urination frequency 4 times. These results indicated that the patient was located in the desmopressin region. He had no severe cardiac dysfunction, renal dysfunction, marked hyperglycemia, or hyponatremia. Oral desmopressin $25 \mu \mathrm{g}$ /day was started, and his daily urine volume, nocturnal urine volume, IPSS score (total and storage), IPSS-Q7 score, and IPSS-QOL score had decreased 8 weeks after the initiation of this treatment, as shown in Figure 2.

\section{Discussion}

In the present study, none of our patients exhibited polyuria with a urine output of more than $50 \mathrm{~mL} / \mathrm{kg} /$ day or 3-4 L/day, which are the diagnostic criteria for DI. However, in some patients, plasma AVP was not sufficiently elevated while plasma osmolality was higher than $290 \mathrm{mOsm} / \mathrm{kg}$ under free water drinking. Therefore, these patients appeared to have a shortage of AVP.
If AVP is low, the reabsorption of water in the renal tubules may be suppressed, causing mild dehydration and a slight increase in serum osmolality. The secretion of AVP is stimulated by an increase in serum osmolality; however, when AVP secretion from the posterior pituitary gland cannot sufficiently cope with the increased serum osmolality, the increase in plasma AVP becomes insufficient. Additionally, when urine output is increased, for example, $30 \mathrm{mg} / \mathrm{kg} /$ day or more, patients may be good candidates for low-dose oral desmopressin administration. The urinary osmolality of patients in the desmopressin region exceeded $300 \mathrm{mOsm} / \mathrm{kg}$ in most cases, and may have been compensatory for the relative AVP shortage due to the overexpression of vasopressin V2 receptors in the renal tubules. ${ }^{9}$

Decreases in the secretion of AVP from the posterior pituitary may be caused by intracranial diseases. In the present study, no significant differences were observed in the frequency of intracranial diseases between patients in the desmopressin and non-desmopressin regions, and the pathological condition directly decreasing AVP secretion 
A

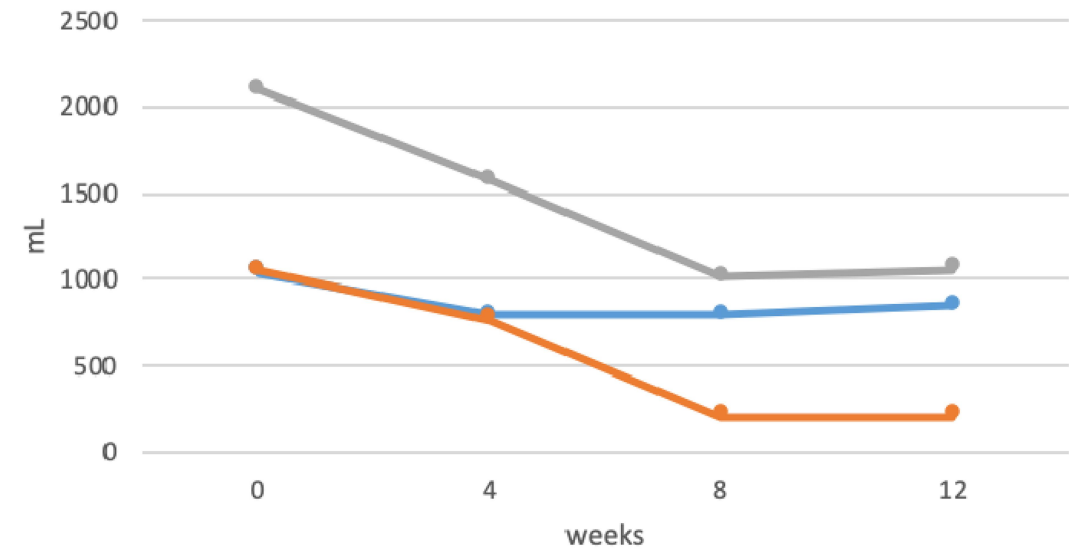

$\Longrightarrow$ daytime urine volume $\longrightarrow$ night time urine volume $\longrightarrow$ 24-hour urine volume

B

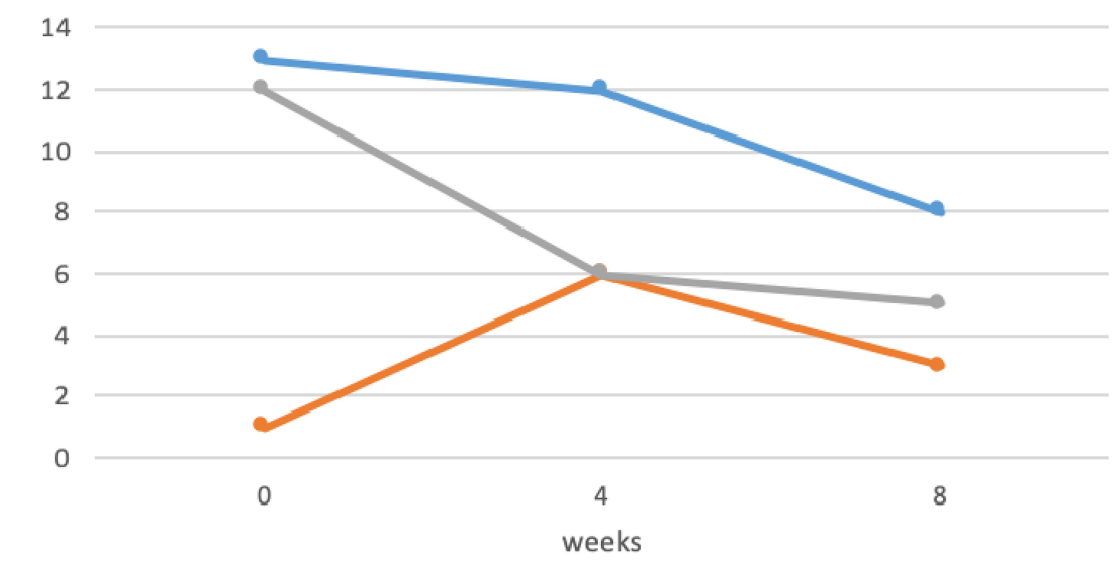

C
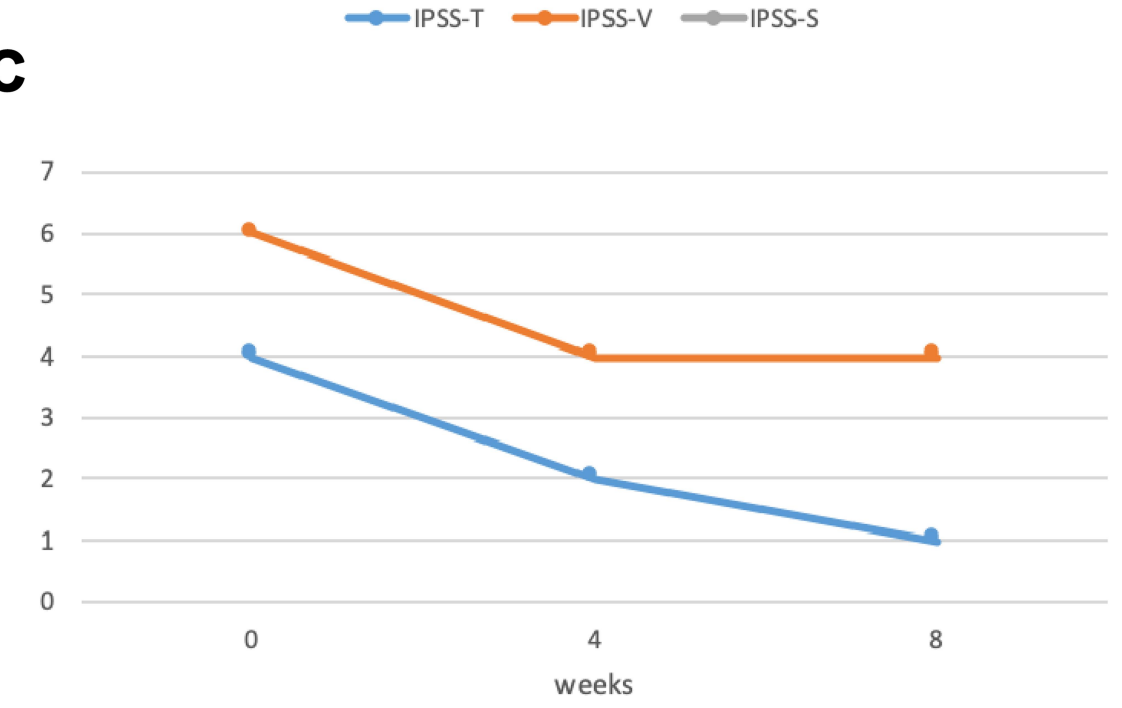

Figure 2 (A) Changes in urine volume following the administration of desmopressin. (B) Changes in IPSS following the administration of desmopressin. IPSS-T: total IPSS score, IPSS-V: IPSS voiding score, IPSS-S: IPSS storage score. (C) Changes in the IPSS-Q7 score and IPSS-QOL score following the administration of desmopressin. 
was not identified. Therefore, decreased AVP secretion appeared to be idiopathic.

The development of polyuria in prominently hyperglycemic patients is due to osmotic diuresis, not a deficiency in AVP. Therefore, it is not a target of desmopressin. In addition, patients with elevated plasma BNP, indicating cardiac dysfunction and/or decreased renal function, need to be excluded because desmopressin may induce heart failure due to water overload.

Polyuria-polydipsia cases with low plasma osmolality and AVP may be caused by an increased water intake. The administration of desmopressin to these cases may induce hyponatremia and heart failure. Therefore, they are not a target for desmopressin. No significant differences were observed in urine volume, urinary frequency, or urination questionnaire scores between patients in the desmopressin and non-desmopressin regions. Therefore, AVP-shortage patients need to be selected for treatment with oral desmopressin based on measurements of serum osmolality and plasma AVP. When desmopressin was administered following the selection of appropriate patients, a reduced urine volume, decreased IPSS and OABSS, improved QOL scores, and no adverse events were observed.

The limitations of this study were that there was an insufficient number of elderly patients with urinary symptoms lacking AVP to prospectively administer low-dose desmopressin, and we were unable to demonstrate a significant advantage of selecting elderly patients lacking AVP.

\section{Conclusions}

After the exclusion of patients with marked hyperglycemia and decreased cardiac or renal function, low-dose oral desmopressin may be administered to patients with increased urine output, nocturia, elevated plasma osmolality, and relatively low plasma AVP.

\section{Funding}

There is no funding for this study.

\section{Disclosure}

The authors report no conflicts of interest for this work or regarding the publication of this study.

\section{References}

1. Robinson AG, Verbalis JG. Posterior pituitary. In: Melmed S, Polonsky K, Larsen RP, Kronenberg H, editors. Williams Textbook of Endocrinology. 13th ed. Amsterdam: Elsevier; 2015:300-320.

2. Gubbi S, Hannah-Shmouni F, Koch CA, Verbalis JG. Diagnostic Testing for Diabetes Insipidus. In: Feingold KR, Anawalt B, Boyce A, et al. editors. Endotext [Internet]. South Dartmouth (MA): MDText.com, Inc; 2000. Accessed https://www.ncbi.nlm.nih.gov/ books/NBK537591/. September 3, 2020.

3. Fenske W, Allolio B. Current state and future perspectives in the diagnosis of diabetes insipidus: a clinical review. J Clin Endocrinol Metab. 2012;97(10):3426-3437. doi:10.1210/jc.2012-1981

4. Fenske W, Quinkler M, Lorenz D, et al. Copeptin in the differential diagnosis of the polydipsia-polyuria syndrome - revisiting the direct and indirect water deprivation tests. J Clin Endocrinol Metab. 2011;96 (5):1506-1515. doi:10.1210/jc.2010-2345

5. Zerbe RL, Robertson GL. A comparison of plasma vasopressin measurements with a standard indirect test in the differential diagnosis of polyuria. New Engl J Med. 1981;305(26):1539-1546. doi:10.1056/ NEJM198112243052601

6. Weiss JP, Herschorn S, Albei CD, van der Meulen EA. Efficacy and safety of low dose desmopressin orally disintegrating tablet in men with nocturia: results of a multicenter, randomized, double-blind, placebo controlled, parallel group study. J Urol. 2013;190 (3):965-972. doi:10.1016/j.juro.2012.12.112

7. Yamaguchi O, Juul KV, Falahati A, Yoshimura T, Imura F, Kitamura M. Efficacy and safety of 25 and $50 \mu \mathrm{g}$ desmopressin orally disintegrating tablets in Japanese patients with nocturia due to nocturnal polyuria: results from two Phase 3 studies of a multicenter randomized double-blind placebo-controlled parallel-group development program. Low Urin Tract Symptoms. 2020;12(1):8-19. doi:10.1111/ luts. 12276

8. Robertson GL. Disorders of the Neurohypophysis. In: Jameson LJ, Fauci AS, Kasper DL, Hauser SL, Longo DL, Loscalzo J. editors. Harrison's Principles of Internal Medicine. Vol. II, 20th. New York: McGraw-Hill Company;2018:2684-2692

9. Thompson CJ. Polyuric states in man. Baillieres Clin Endocrinol Metab. 1989;3(2):473-497. doi:10.1016/S0950-351X(89)80012-6

\section{Publish your work in this journal}

Research and Reports in Urology is an international, peer-reviewed, open access journal publishing original research, reports, editorials, reviews and commentaries on all aspects of adult and pediatric urology in the clinic and laboratory including the following topics: Pathology, pathophysiology of urological disease; Investigation and treatment of urological disease; Pharmacology of drugs used for the treatment of urological disease. The manuscript management system is completely online and includes a very quick and fair peer-review system, which is all easy to use. Visit http://www.dovepress.com/ testimonials.php to read real quotes from published authors. 